\title{
Characterization of PM10 Particles by SEM-EDS
}

\author{
R. Ramirez-Leal ${ }^{1}$, M. Cruz-Campas ${ }^{1}$ and H. Estuardo-Moreno ${ }^{1}$
}

1. Sonora State University. Ley Federal del Trabajo s/n Col. Apolo, C.P.; Hermosillo, Sonora, Mexico.

The particles that are normally suspended in urban atmospheres, are of natural origin or produced by human activities. These can be introduced into the air as solid or liquid particles or may be formed by photochemical reactions from contaminants already present in the air [1].

Depending on their size, the particles can float or sediment. Particles that remain floating are known as total suspended particles or TSP. Particles whose diameter is less than or equal to $10 \mu \mathrm{m}$ are known as inhalable fraction particles or PM10, which may be formed by aerosols, powders, metals, combustion products, or microorganisms such as protozoa, bacteria, viruses, fungi and pollen that can cause different types of diseases. When particles are inhaled they are not always expelled by the body's defense systems, causing problems in the respiratory system.

Exposure to PM10 has been a major concern in recent years, as studies showing a significant association between the environmental concentration of the respirable fraction particles and the mortality and morbidity of the population are more frequent. Consistently across many studies a $3 \%$ increase in normal daily mortality for every $10 \mu \mathrm{g} / \mathrm{m} 3$ in PM10 has been found from the value of the standard. It is the most significant association with cardiopulmonary and lung cancers [2].

The aim of this work is identification and characterization (morphology and chemical composition) of particles present in samples of PM10 as part of an air quality study by SEM-EDS. The City of Hermosillo is located between latitude $20^{\circ} 01^{\prime} 00$ "and $20^{\circ}$ 08' 30" north latitude and between the meridian $110^{\circ} 54^{\prime} 30$ "and $111^{\circ} 01^{\prime} 00^{\prime \prime}$ west longitude at an altitude of $200 \mathrm{~m}$ above sea level; is the Sonora state's capital, located to the Center-West of the coastal plain, in northwestern Mexico [3].

The PM10 particles was collected by high-volume sampler, located in the northeast of the city, using a sampling flow of $1.3 \mathrm{~m} 3 / \mathrm{min}$. Sampling was carried for $24-\mathrm{h}$ one day a week. The filters used were quartz, with size of $8 \times 10$ inch that captured PM10 particles. Analysis of PM10 were performed using scanning electron microscopy (SEM) JEOL Model JSM-5800LV coupled with energy dispersive spectrometer (EDS) EDAX DX prime brand, for determination of morphology and elemental composition of airborne particles. Specimens were processed by separating the collected particles from the quartz filters by means of submersing a $2 \mathrm{~cm} 2$ section of each filter into isopropilic alcohol within a test tube for 5 minutes. Then, an aliquot of the suspension was placed over a sample holder, and is introduced into the chamber of SEM.

It is observed that the morphology of the particles is irregular, rough, smooth and with agglomerates.(Fig. 1) The studies by EDS to PM10 showed in monitoring station, that elements are: Al (At: 33.8), Si (At: 14,43), Na (At: 00.893), Ca (At: 00.745), Mg (At: 01.38), Ti (At: 02.25) and Fe (At: 04.157). (Fig. 2) The results obtained from the characterization of the particles captured showed that elements such as $\mathrm{Al}, \mathrm{Si}, \mathrm{Ca}$, and $\mathrm{Na}$ are usually related with litophilic sources; other chemical elements such as $\mathrm{Fe}$ are more commonly related various human activities. The elements found in the filters of PM10 indicate the importance of the individual characterization of atmospheric aerosols, because of the 
limited amount of information on the speciation and size of atmospheric particles. Additionally, the identification of the morphology and chemical composition of these particles provides valuable information for the determination of their origin. The chemical analyses performed on the aerosol forming conglomerates allow us to determine air quality on each city. However, these might ignore the chemical elements that occur in smaller amounts because these are not considered or detected during the elaboration the laws established according to normal determinations.

References:

[1] Ma. Cristina Castañón Bautista et al, International Journal of Applied Science and Technology 5 (2) (2015).

[2] Yunbo Zhai, Zongmin Fu and Lafang Wang, Environ Monit Assess 184 (2012), p. 6693.

[3] R. Ramirez-Leal, Microsc. Microanal. 15 (Suppl. 2) (2009) p. 1300.
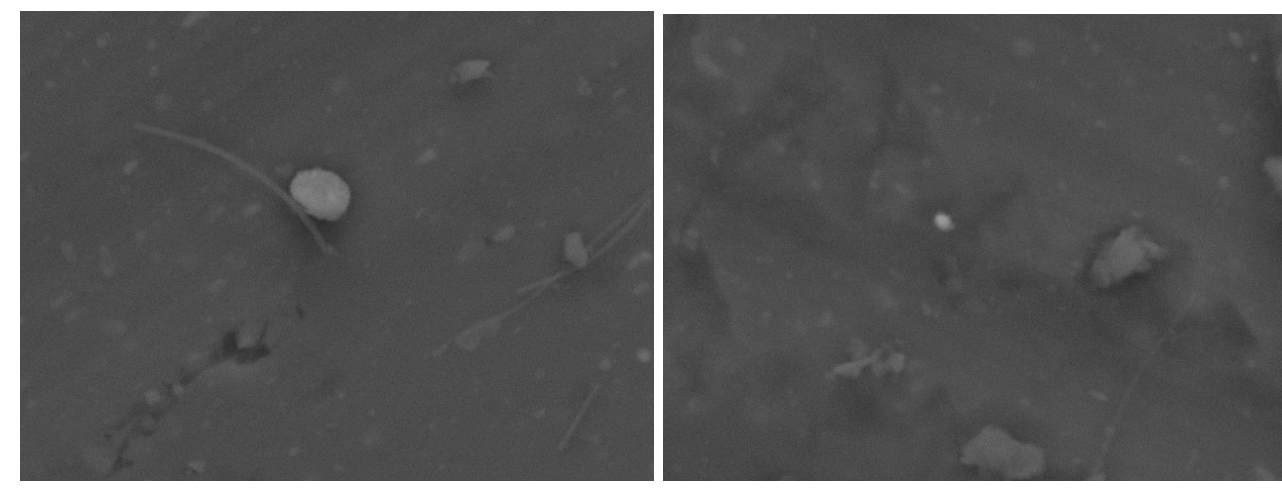

Figure 1. Micrograph of the sample in SEM-EDS. 5000x BEI and $15 \mathrm{kV}$

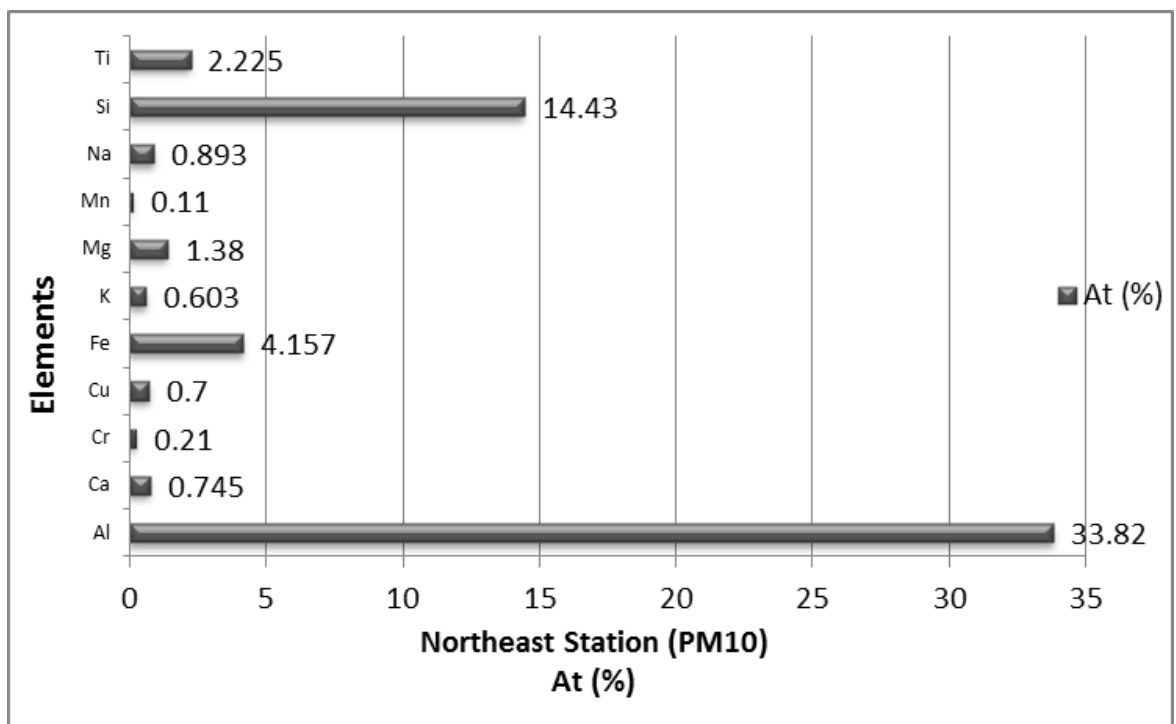

Figure 2. Majority of elemental constituents of PM10 samples in northeast area (Averaged values of atomic percentage (At\%) for all filter samples). 